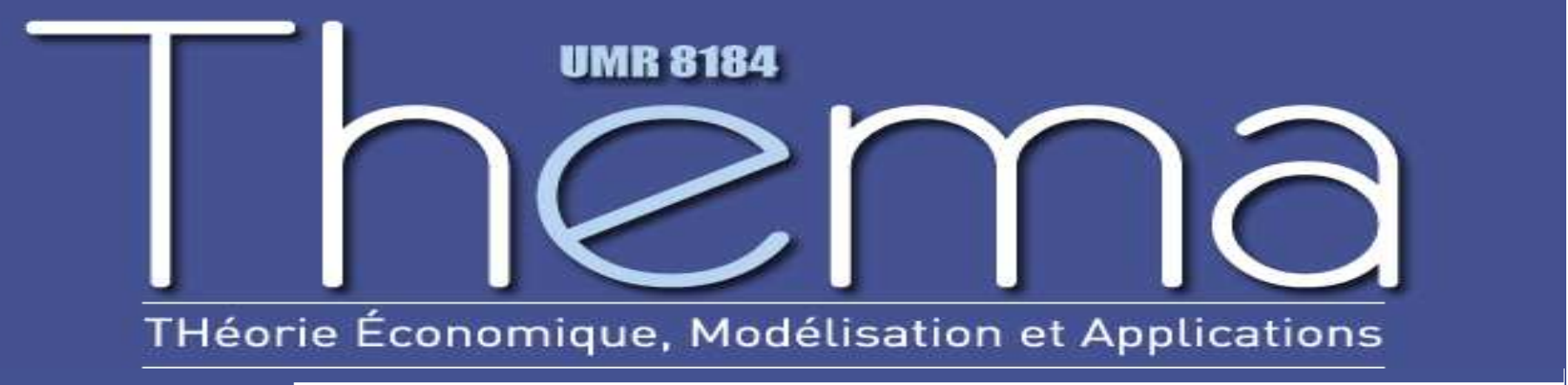

THEMA Working Paper n²015-02 Université de Cergy-Pontoise, France

\title{
Uncertain discount and hyperbolic preferences
}

Daniele Pennesi

Janvier 2015 


\title{
Uncertain discount and hyperbolic preferences
}

\author{
Daniele Pennesi*
}

January 2015

\begin{abstract}
When the discount rate is uncertain, individuals whose preferences are consistent with discounted expected utility, exhibit diminishing impatience. This paper introduces and characterizes a variation of discounted expected utility in which the discount rate depends on the state of the nature that will occur. Quasi-hyperbolic discounting and the model of Dasgupta and Maskin (2005) are particular cases. The present bias disappears when the immediate payoff becomes uncertain. Commitment may be detrimental unless the discount rate is constant.
\end{abstract}

KEYWORDS: Diminishing Impatience, Uncertainty, Hyperbolic Discounting, Time Inconsistency

Jel Classification: D03, D90, D81

*THÉMA (University of Cergy-Pontoise). Email: daniele.pennesi@u-cergy.fr This research was supported by Labex MMEDII (ANR-11-LBX-0023-01). 


\section{Introduction}

Economists and psychologists often assume that individuals are impatient. The utility of a reward decreases as the time before its experience increases. Impatience is typically modelled using a constant discount rate compounded over time (Koopmans, 1960). However, experimental evidence ${ }^{1}$ suggests that actual discount rates are not constant, but they decline over time: the further away is a payoff, the higher is individual's patience. Such phenomenon is called Diminishing Impatience (DI), it is a source of dynamic inconsistency and it is not compatible with the standard exponential discounting. The most popular explanation for DI is lack of self control (Laibson, 1997), the temptation to consume is higher when the consumption is closer in time. This paper shows that, under uncertainty, a simple generalization of the discounted expected utility model accounts for diminishing impatience. Intuitively, uncertainty about the true state of the world may affect the time preferences of the individual, in particular, the discount rate used to evaluate future utilities attached to the state. Before uncertainty is resolved, the individual is unsure about the discount rate she will use, even when evaluating certain payoffs. Such an uncertainty is sufficient to generate diminishing impatience. When uncertainty is resolved, the individual discounts future utility exponentially, according to the discount rate attached to the realized state. Formally, the Uncertain Discount Expected Utility (UDEU) model evaluates an uncertain consumption plan $c: \Omega \rightarrow \mathbb{R}^{\mathbb{T}}$ (a map from states of nature to consumption streams) by:

$$
V(c)=E_{p}\left[\sum_{t=0}^{T} \delta_{\omega}^{t} u(c(\omega, t))\right]
$$

where $\omega \in \Omega$ is a (Savagean) state of nature and $p$ is a subjective probability. The model can be interpreted in the following sense: the individual fixes a state $\omega$, she calculates the present value of the consumption stream $(c(\omega, 0), c(\omega, 1), \ldots, c(\omega, T))$ according to the utility $u$ over the payoffs and the discount rate $\delta_{\omega}$. She repeats the same procedure for all states and she aggregates the present values using the subjective probability $p$. When evaluating future rewards that do not depend on the state of the world, the actual discount rate is the expected value of the discount rates $E_{p}\left[\delta_{\omega}^{t}\right]$. Diminishing impatience occurs since, as the delay increases, the more patient rates decrease more slowly than the less patient ones, lowering the overall impatience (Theorem 3). There are two important special cases

\footnotetext{
${ }^{1}$ See Thaler (1981); Benzion et al. (1989).
} 
of UDEU: the quasi-hyperbolic discounting model of Laibson (1997), who corresponds to UDEU with only two distinct discount rates, one of which is extremely impatient (see Sec. 5.1). The second case is the model of Dasgupta and Maskin (2005), where they shows that uncertainty about the actual realization of a payoff explains the preference reversal often ascribed to DI. Their model corresponds to UDEU where the states of nature are dates as well, i.e. $\Omega=\mathbb{T}$. It represents an individual who has a dual time schedule, one physical and one subjective (see Sec. 5.2). The former is the time at which the payoff is supposed to be payed, the latter embodies the subjective possibility of early or late realization. An element of the state space $(s, t)$ is, therefore, interpreted as "the payoff at time $t$ will materialize at time $s^{\prime \prime}$.

Differently from intertemporal choice under certainty, in UDEU the source of DI is the subjective uncertainty about the discount rate that follows from a specific form of statedependent preferences. It is not difficult to argue the plausibility of state dependent preferences, differently, their exponential discount form $\delta_{\omega}^{t} u(c(\omega, t))$ may be questioned. Firstly, the current work contributes to a literature who explains diminishing impatience exploiting the interaction between time preferences and uncertainty. ${ }^{2}$ Since we are assuming that uncertainty is the only driving force behind diminishing impatience, it is natural to posit that absence of uncertainty or the knowledge of the true state of the world should make the individual dynamically consistent. This is true in UDEU due to exponential discounting of conditional preferences. If the individual knows the true state of the world $\hat{\omega}$, she will evaluate future consumption using $\delta_{\hat{\omega}}^{t} u(c(t, \hat{\omega}))$, a dynamically consistent rule. In other words, if the state of the world affects the individual's tastes about future consumption, when knowing the true state she can anticipate her future tastes and she should behave consistently. Second, from a descriptive point of view, we would like to replicate, as a thought experiment (see Section 6), the experimental result of Keren and Roelofsma (1995). They argue that the main difference between the "present" and the "future" is the intrinsic uncertainty of the latter. Indeed, when they introduced uncertainty in the immediate payoff they observe a drastic reduction of the present bias. ${ }^{3}$ In UDEU, we can observe a similar behavior if we introduce a precise form of uncertainty in the immediate payoffs (see Fact 1).

The interaction between time and uncertainty as a possible explanation for diminishing

\footnotetext{
${ }^{2}$ Sozou (1998), Azfar (1999), Dasgupta and Maskin (2005), Halevy (2008), Farmer and Geanakoplos (2009) and Epper and Fehr-Duda (2014)

${ }^{3}$ The present bias is a particular case of DI, in which the impatience is greatest at the present.
} 
impatience, has been already proposed, under various forms, in the literature. The main novelty of the present work is the fully subjective foundation for uncertainty of the future. Indeed, in all the existing models, uncertainty is assumed exogenously, either through an explicit hazard rate governing the probability of experiencing future utility or, directly assuming an uncertain discount rate. However, a more natural approach would involve subjective uncertainty of the future, (as noted in Halevy, 2008). Indeed, the uncertainty of the future can be justified by the Implicit Risk approach that "associates delayed consequences to a subjective implicit risk" (Halevy, 2008, p. 1146). In our model uncertainty is fully subjective since it follows from a subjective interaction between states of the world and time preferences.

Understanding the nature of dynamic inconsistency is also important to set up commitment devices to prevent the inconsistency. With UDEU, we derived a negative result (Theorem 5): commitment before uncertainty is resolved is always valuable, if and only if, there is no uncertainty about the discount rate. In other words, unless the individual has a standard discounted expected utility preference, she may want to change her choice after the true state of the world is realized. The result raises the question of how to design effective commitment devices under uncertainty.

The model also offers a behavioral foundation for the discount of "far future" introduced by Weitzman (1998). He claims that, under uncertainty of the discount rate, it is more natural to averages the discount factors, i.e. $E\left[\tilde{\delta}^{t}\right]$ rather than the discount rates $E[\tilde{\delta}]^{t}$. Our model can be interpreted as the utility of a planner that evaluates the present value of future payoffs (environmental policies) when the discount is uncertain.

\section{An introductory example}

The following is a variation of the bird example described in Dasgupta and Maskin (2005).

Suppose a bird is waiting some berries to ripen. There are two bushes of berries. One bush produces smaller berries that will ripen in 2 days, the other produces bigger berries, but they take 7 days to ripen. Each bush produces berries once a month (of 28 days). The payoffs are then, $(b, 2)$ vs $(B, 7)$ and $(b, 30)$ vs $(B, 35)$. Suppose you want to know the current preference of the bird for the two bushes. The bird, however, is uncertain about the actual distance between his nest and the bushes. There are two possibilities, each bush can be 
nearby or far away. The states of the world are $\Omega=\{\mathrm{nn}, \mathrm{nf}, \mathrm{fn}, \mathrm{ff}\}$. The bird considers the four states equally likely, so each one has a probability $p=0.25$. The uncertainty is resolved only after the bird chooses a bush to pick berries. We assume that the distance affects the time preferences of the bird, for example, if both bushes are far away the present value of the berries is lower because of the greater cost of reaching the bushes. Next table sums up the information about probability and the discount rates attached to each state.

\begin{tabular}{c|cccc} 
& $\mathrm{nn}$ & $\mathrm{nf}$ & $\mathrm{fn}$ & $\mathrm{ff}$ \\
\hline $\mathrm{p}$ & 0.25 & 0.25 & 0.25 & 0.25 \\
$\delta$ & 0.99 & 0.9 & 0.9 & 0.7
\end{tabular}

The distance between the nest and the bushes does not affect the payoffs (the berries), therefore, the bird is asked to rank the following pairs of alternatives:

\begin{tabular}{c|crc|c}
$(b, 2)$ & $\mathrm{t}=2$ & & $(B, 7)$ & $\mathrm{t}=7$ \\
\cline { 1 - 2 } $\mathrm{nn}$ & $b$ & & $\mathrm{nn}$ & $B$ \\
$\mathrm{nf}$ & $b$ & vs & $\mathrm{nf}$ & $B$ \\
$\mathrm{fn}$ & $b$ & & $\mathrm{fn}$ & $B$ \\
$\mathrm{ff}$ & $b$ & & $\mathrm{ff}$ & $B$
\end{tabular}

\begin{tabular}{c|cccc|c}
$(b, 30)$ & $\mathrm{t}=30$ & & $(B, 35)$ & $\mathrm{t}=35$ \\
\cline { 1 - 2 } $\mathrm{nn}$ & $b$ & & $\mathrm{nn}$ & $B$ \\
$\mathrm{nf}$ & $b$ & vs & $\mathrm{nf}$ & $B$ \\
$\mathrm{fn}$ & $b$ & & $\mathrm{fn}$ & $B$ \\
$\mathrm{ff}$ & $b$ & & $\mathrm{ff}$ & $B$
\end{tabular}

Assume $u(b)=5$ and $u(B)=7$, simple calculations ${ }^{4}$ show that $(b, 2)>(B, 7)$ and $(b, 30) \prec$ $(B, 35)$. Today, the bird prefers the smaller/earlier berries to the larger/later ones, but it reverses its preferences when asked to rank bushes for the next ripening cycle. This is the preference reversal often ascribed to diminishing impatience. However, it follows from uncertainty of the discount rate, rather than lack of self control or uncertainty about the actual realization of the payoffs.

In addition, we claim that uncertainty about the distance between the nest and the bushes is the only reason for exhibiting diminishing impatience. If the bird knew the actual distance of the two bushes, it would be normatively sound to observe a dynamically consistent choice. Indeed, suppose "fn" is the true state and it is known by the bird, it follows that $(b, 2)>(B, 7)$ and $(b, 30)>(B, 35)$, the preference of a bird who discounts the future exponentially.

\subsection{Related literature}

The possibility that individuals may behave inconsistently over time has been recognized by Strotz (1956) and supported experimentally by Thaler (1981) and Benzion et al. (1989)

\footnotetext{
${ }^{4} u(b)\left(0.25 \times 0.99^{2}+0.5 \times 0.9^{2}+0.25 \times 0.7^{2}\right)>u(B)\left(0.25 \times 0.99^{7}+0.5 \times 0.9^{7}+0.25 \times 0.7^{7}\right)$ and $u(b)\left(0.25 \times 0.99^{30}+\right.$ $\left.0.5 \times 0.9^{30}+0.25 \times 0.7^{30}\right)<u(B)\left(0.25 \times 0.99^{35}+0.5 \times 0.9^{35}+0.25 \times 0.7^{35}\right)$
} 
among the others. For a review Shane et al. (2002). Dynamic inconsistency in the form of diminishing impatience can be rationalized using the hyperbolic discounting model of Loewenstein and Prelec (1992). A more parsimonious model is the quasi-hyperbolic or $\beta$ - $\delta$ model of Laibson (1997), who assumes diminishing impatience only between the present and the future. Axiomatic foundations for quasi-hyperbolic preferences are provided by Hayashi (2003) and Montiel Olea and Strzalecki (2014). Hyperbolic and quasihyperbolic discounting do not cope with uncertainty. Harris and Laibson (2013) introduced the Present-Future model, a stochastic extension of the subjective discount model of Luttmer and Mariotti (2003), in which uncertainty concerns the duration of the "present" and the Instantaneous Gratification model, a limiting case of the Present-Future model. However, they do not provide any behavioral foundation.

The present work contributes to a different strand of literature who exploits the interaction between time preferences and uncertainty to explain diminishing impatience. The consideration that any future payoff is intrinsically uncertain dates back to Fisher (1930) and Yaari (1965). Mortality risk, for example, invariably affects any future payoff. This intuition is supported by a large body of experimental evidence (Keren and Roelofsma (1995), Weber and Chapman (2005) and Myerson et al. (2003)) showing similarities between discounting and uncertainty. Uncertainty of the future has been modelled in different ways: Sozou (1998) assumes the existence of an uncertain hazard rate governing the probability of experiencing future utility to rationalize diminishing impatience. Dasgupta and Maskin (2005) introduces a different form of uncertainty that does not affect the hazard rate, but the timing at which payoffs are realized, the relation with UDEU is highlighted in Section 5.2. Differently, Azfar (1999) and Farmer and Geanakoplos (2009) rationalize diminishing impatience directly assuming uncertainty in the discount rate. Both of them assume exogenous uncertainty of the discount rate without providing foundation for this assumption. It is worth noticing that, the type of uncertainty in Sozou (1998) and Dasgupta and Maskin (2005) is not distinguishable from uncertainty of the discount rate as in Azfar (1999) and Farmer and Geanakoplos (2009), therefore, our model provides a behavioral characterization for both. To the best of our knowledge, Higashi et al. (2009) is the only work with an axiomatization of subjectively random discount rates, however, their primitive is a preference over menus of objective lotteries.

An elegant alternative involving uncertainty of the future has been proposed by Halevy 
(2008), Saito (2011) and Epper and Fehr-Duda (2014). They completely identify intertemporal choice with choice under uncertainty, any future payoff runs the risk of disappearing according to a hazard rate. Differently from Sozou (1998), the hazard rate is known, but it is distorted by a weighting function similar to the one of the rank-dependent utility. DI and others anomalies of intertemporal choice may be explained imposing conditions on the curvature of the distortion function. With respect to them, our model may explain DI without relying on distortion functions, in addition, we characterize "uncertainty of the future" in a subjective fashion, whereas they introduce uncertainty exogenously. An application of uncertainty of the discount rate to an overlapping generation model can be found in Karni and Zilcha (2000). In a different domain, Jackson and Yariv (2012) obtain a result, similar to Theorem 3, as a consequence of aggregation of intertemporal preferences of individuals with different discount rates.

\section{Axiomatic foundation of UDEU}

UDEU is a simple modification of discounted expected utility so, its axiomatic foundation is a straightforward generalization of that of EU. First, notice that any choice over uncertain consumption streams can be seen as a choice over acts on a product space, where the two factors are the states of the world $\Omega$ and time. Hence, an element of the state-space is a pair $(\omega, t)$ and it uniquely identifies a payoff, the payment delivered at time $t$ in state $\omega$. The intuition behind the axiomatization is that, unless explicitly imposed through axioms, any expected utility model over a product space allows for subjective correlation between the two "sources of uncertainty". In this case, we assume that the state of nature affects time preferences. This translates into a state-dependent intertemporal utility. Imposing a standard stationarity axiom on conditional preferences will give a state-dependent discount rate.

Time is discrete and finite, $t \in \mathbb{T}$ and $\mathbb{T}=\{0, \ldots, T\}$. We assume the existence of a finite set $\Omega$, containing the states of the world with $|\Omega|>2$. An act is a function $h: \Omega \times \mathbb{T} \rightarrow$ $[w, b]$ for some bounded interval $[w, b] \subset \mathbb{R}$, the set of acts is denoted $\mathscr{H}$. The individual has a preference $\succcurlyeq$ over acts. We assume that uncertainty resolves immediately after a choice is made and payoffs are realized contextually, so that at time zero consumption may be uncertain but it is not discounted. This assumption is not fundamental, however, it is 
employed in Section 6. Finiteness of the time horizon and of the states of the world, allows to identify any act $h$ with a matrix:

\begin{tabular}{c|cccc}
$\Omega \times \mathbb{T}$ & 0 & 1 & $\ldots$ & $\mathrm{T}$ \\
\hline$\omega_{1}$ & $h\left(\omega_{1}, 0\right)$ & $h\left(\omega_{1}, 1\right)$ & $\ldots$ & $h\left(\omega_{1}, T\right)$ \\
$\omega_{2}$ & $h\left(\omega_{2}, 0\right)$ & $h\left(\omega_{2}, 1\right)$ & $\ldots$ & $h\left(\omega_{2}, T\right)$ \\
$\vdots$ & $\ldots$ & $\ldots$ & $\ldots$ & $\ldots$ \\
$\omega_{N}$ & $h\left(\omega_{N}, 0\right)$ & $h\left(\omega_{N}, 1\right)$ & $\ldots$ & $h\left(\omega_{N}, T\right)$
\end{tabular}

If an act is independent of the state, i.e. $h(\omega, \cdot)=h\left(\omega^{\prime}, \cdot\right)$ for all $\omega, \omega^{\prime} \in \Omega$, we call it a stateindependent act and we, sometime, denote it as $h=(x, y, \ldots, z)$. If it also independent of the time, i.e. $h(\omega, t)=h\left(\omega^{\prime}, t^{\prime}\right)$ for all $(\omega, t) \in \Omega \times \mathbb{T}$, we call it a constant act, and we identify it as an element of $[w, b]$. We write $(x, t)$, the act $h$ defined as: $h(\cdot, t)=x$ for all $\omega \in \Omega$ and $h(\omega, s)=z$ with $u(z)=0$ for all $\omega \in \Omega$ and $s \neq t$, it represents a sure payment $x$ at time $t$. A state $(\omega, t)$ is null if $x_{\omega, t} f \sim f$ for all $x \in[w, b]$ and $f \in \mathscr{H}$.

The first axiom implies a minimal representation of preferences over acts:

Axiom (Utility). $\succcurlyeq$ is represented by a continuous and monotone increasing non-constant function $V$.

Given $(\omega, t) \in \Omega \times \mathbb{T}$, we denote $f_{\omega t} g$, the act giving $f$ at time $t$ and state $\omega$ and $g$ otherwise. The next axiom is the trade-off consistency of Köbberling and Wakker (2003) and it is necessary and sufficient to characterize an "expected utility" representation over the product state-space $\Omega \times \mathbb{T}$ :

Axiom (T-OC). For any acts $f, g, h, h^{\prime} \in \mathscr{H}$ and any $\omega, \omega^{\prime} \in \Omega$ and $t, t^{\prime} \in \mathbb{T}$,

$$
x_{\omega t} f \sim y_{\omega t} g, \quad x_{\omega t}^{\prime} f \sim y_{\omega t}^{\prime} g, \text { and } x_{\omega^{\prime} t^{\prime}} h \sim y_{\omega^{\prime} t^{\prime}} h^{\prime} \Longrightarrow x_{\omega^{\prime} t^{\prime}}^{\prime} h \sim y_{\omega^{\prime} t^{\prime}}^{\prime} h^{\prime}
$$

We now define $\Omega$-conditional preferences and we write $f \succcurlyeq_{\omega} g$, if and only if, $f_{\omega} h \succcurlyeq g_{\omega} h$ for some $h$. By T-OC they do not depend on $h$. Next axiom involves conditional preferences only and it establishes a conceptual distinction between the states of the world and time. Indeed, up to now, we treated the two halves of the state space, states of nature and dates, symmetrically. However, it is clear that the time's arrow has a precise direction. The next axiom restores the desired conceptual separation. It imposes standard geometric discounting conditionally on each state in $\Omega$. 
Axiom (Conditional Stationarity). For all state-independent act $\left(x_{0}, x_{1}, \ldots, x_{T}\right) \in \mathscr{H}, x, y, x^{\prime}, y^{\prime} \in$ $[w, b]$ and $\omega \in \Omega$, if

$$
\left(x_{0}, x_{1}, \ldots, x_{t-1}, x, x^{\prime}, x_{t+2}, \ldots\right) \succcurlyeq_{\omega}\left(x_{0}, x_{1}, \ldots, x_{t-1}, y, y^{\prime}, x_{t+2}, \ldots\right)
$$

for some $t, t+1$, then it is true for all $t \in \mathbb{T}$.

Axiom Conditional Stationarity postulates that, conditionally on the realization of a state of the world, preferences over state-independent acts are stationary. This is the axiom that discriminates UDEU from discounted expected utility. The reasons why we posit Conditional Stationarity are both normative and descriptive. From the normative point of view, we believe that the uncertainty of the future is the only force driving diminishing impatience. Therefore, once uncertainty is resolved or if there is no uncertainty at all, the individual should behave consistently. From the descriptive point of view, we would like to replicate in our setting, the results of Keren and Roelofsma (1995) (see Section 6). Introducing uncertainty in the immediate reward, forces an UDEU individual to discount geometrically, so to behave in a dynamically consistent fashion. This is similar to the results found experimentally by Keren and Roelofsma (1995), in which the introduction of objective uncertainty in the immediate reward, drastically decreases the percentage of subjects exhibiting the present bias.

Turning to the representation theorem, standard arguments lead to the following result:

Theorem 1. Suppose there are at least two non-null states. Axioms Utility, T-OC and Conditional Stationarity are satisfied, if and only if, there exists a utility $V$ representing $\succcurlyeq$ of the form

$$
V(h)=\sum_{\omega \in \Omega} p(\omega) \sum_{t \in \mathbb{T}} \delta_{\omega}^{t} u(h(t, \omega))
$$

where $p$ is a unique probability, $\delta_{\omega}>0$ for all $\omega \in \Omega$ and $u$ is a strictly increasing and cardinally unique utility function.

UDEU is a minimal generalization of discounted expected utility taking into account the possibility that uncertainty affects pure time preferences. 


\section{Diminishing impatience}

In this section we show how UDEU can explain diminishing impatience (or hyperbolic preferences) and, in particular, the present bias. We use the definition given in Prelec (2004):

\section{Definition 2.}

DI. $\succcurlyeq$ exhibits diminishing impatience, iffor any $y>x>0$,

$$
(x, t) \sim(y, t+1) \text { implies }(x, t)>(y, t-1)
$$

for all $0<t \leq T-1$.

PB. $\succcurlyeq$ exhibits the present bias, iffor any $y>x>0$,

$$
(x, t) \sim(y, t+1) \text { implies }(x, 0)>(y, 1)
$$

for all $0<t \leq T-1$.

As the payment is delayed, according to DI, the bigger payoff becomes more attractive than the smaller one. The present bias is the particular case of DI at 0 . When discounting is hyperbolic as in Loewenstein and Prelec (1992) and the utility and the discount function are separated, a preference exhibits DI, whereas quasi-hyperbolic discounting of Laibson (1997) accounts for the PB but not for DI.

We can equivalently define DI in terms of an impatience index. Assuming $V\left(x_{1}, \ldots, x_{T}\right)=$ $\sum_{t} d(t) u\left(x_{t}\right)$, with $d(0)=1$. Let $I(t)=\frac{d(t)}{d(t+1)}$ then,

- DI is equivalent to $I(t)>I(t+1)$ for all $0 \leq t \leq T-1$

- $\mathrm{PB}$ is equivalent to $I(0)>I(t)$ for all $0<t \leq T-1$

Next result shows that UDEU exhibits DI:

Theorem 3. Let $I(t)=\frac{E_{p}\left[\delta_{\omega \omega}^{t}\right]}{E_{p}\left[\delta_{\omega}^{t+1}\right]}$, there exists $\delta_{\omega} \neq \delta_{\omega^{\prime}}$ with $p(\omega)>0, p\left(\omega^{\prime}\right)>0$, if and only if, $\succcurlyeq$ exhibits diminishing impatience.

$\mathrm{As}^{5}$ the time horizon increases, the more patient rates decline more slowly than the less patient ones, lowering the overall impatience. Therefore, diminishing impatience and, in

\footnotetext{
${ }^{5} \mathrm{~A}$ proof can be found in Jackson and Yariv (2012).
} 
particular, the present bias can be explained through a state-dependent discount rate and without assuming any exogenously given probability or psychological explanations. Diminishing impatience is the natural consequence of uncertainty in intertemporal choice.

\section{Special cases}

Two important special cases of UDEU are the quasi-hyperbolic discounting model of Laibson (1997) and the model of Dasgupta and Maskin (2005).

\subsection{Quasi-hyperbolic discounting}

In the quasi-hyperbolic discounting model, the discount rate is equal to 1 at time $t=0$ and to $\beta \delta^{t}$ at time $t>0$ for some $\beta \in[0,1]$. It can be seen as a particular case of UDEU when there are only two distinct discount rates, call them $\delta$ and $\epsilon$, with the second very close to zero (or even equal to zero if we define $0^{0}=1$ ). In that case, when evaluating stateindependent act, we have $E_{p}\left[\delta_{\omega}^{t}\right]=p \delta^{t}+(1-p) \epsilon^{t}$. It corresponds to

$$
E_{p}\left[\delta_{\omega}^{t}\right]= \begin{cases}1 & t=0 \\ p \delta^{t}+(1-p) \epsilon^{t} \approx p \delta^{t} & t>0\end{cases}
$$

For $t>0, E_{p}\left[\delta_{\omega}^{t}\right] \approx p \delta^{t}$, since for $\epsilon$ small enough the second term is negligible or equal to zero if $0^{0}=1$. Quasi-hyperbolic discounting is, then, an extreme case of UDEU, where the individual is completely impatient in a given state (or event) and has the same discount rates in all the remaining states (or events). In UDEU, the $\beta-\delta$ discount is actually a $p-\delta$.

\subsection{Berries and crows}

Dasgupta and Maskin (2005) studies a dynamic preference reversal often ascribed to hyperbolic discounting. Differently from the static situation described above, the individual faces the same choice problem at different points in time. The preference reversal occurs if she changes her mind as time goes by. For example, at time zero she may prefer receiving $(x, t)$ to $\left(y, t^{\prime}\right)$ with $x<y$ and $t<t^{\prime}$, but she reverses preferences at time $s$ with $0<s<t, t^{\prime}$. Dasgupta and Maskin (2005) offers an "evolutionary" explanation, arguing that uncertainty of the payoff combined with a subjective uncertainty concerning the timing of the payoff's 
realization, may explain such a reversal. Our introductory example was built on their anecdotal bird-berries example. They consider a bird waiting some berries to ripen. There are two bushes of berries, with different "utility" and ripening time. The hazard rate at which the berries disappear depends on a flock of crows hanging around who do not care about ripeness. In addition to this, the bird is unsure about the effective timing of ripening, it knows that the berries will ripen at time $t$, but it allows the possibility of an earlier or later ripening. In this sense, the bird acts "as if" there are two time dimensions: one objective, the berries will ripen at time $T$ and the other subjective, the berries will ripen earlier or later. In a continuous time setting they claim that at time zero the bird should evaluate the utility of berries ripening at time $T$ according to:

$$
V(k)=\left[\int_{0}^{T} p e^{-r s} d s+(1-p T) e^{-r T}\right] u(k)
$$

where $r$ is the hazard rate (of crows' arrival), $p$ is the instantaneous probability of early/late realization and $u(k)$ the utility. $p T$ is the probability of realization prior to time $T$.

The discrete time version of Equation (2) is a particular case of UDEU if we assume $\Omega=\mathbb{T}$. The individual acts "as if" there are two different timing for the experience of future utility. One is the physical time at which the reward is given, for example a payment is promised at time $T$, the other is the "subjective timing" representing the possibility of early/late payment. The interpretation of an element of the space $(s, t)$ is "the payoff at time $t$ will materialize at time $s "$.

Given this assumption, a prospect of the type $c=(0,0, \ldots, k)$ with $u(0)=0$ and $k \in \mathbb{R}$ is evaluated by UDEU as:

$$
V(c)=\sum_{s \geq 0} p(s) \delta_{s}^{T} u(k)
$$

Letting $\delta_{s}^{T}=(1-r)^{s} \hat{\delta}^{s}$ for some $\hat{\delta} \in(0,1)$ and $r \geq 0$, we have a discrete version of MaskinDasgupta preferences given by

$$
V(c)=\sum_{s=0}^{T} p(s)(1-r)^{s} \hat{\delta}^{s} u(k)
$$

or

$$
\left[\sum_{s=0}^{T-1} p(s)(1-r)^{s} \hat{\delta}^{s}+p(T)(1-r)^{T} \beta^{T}\right] u(k)
$$

where the time preference are given by the pure time preference $\delta$ and the hazard rate $r$ 
(the crows' arrival). Whereas, the probability $p(s)$ corresponds to the probability of early (or late) realization of the payoff.

\section{Testable predictions of UDEU}

As hinted in the introduction, the structure of UDEU is meant to perform a thought experiment that resembles the experiment of Keren and Roelofsma (1995). They show, in an objective risk setting, that the present bias decreases drastically when the immediate reward becomes risky.

They offered to subjects the following choices over sooner-smaller/later-larger rewards (amounts are in guilders):

Choice 1. 100 now or 110 in 4 weeks

Choice 2. 100 in 26 weeks or 110 in 30 weeks

An individual who chooses 100 now in Choice 1. and for 110 in Choice 2. exhibits the present bias. In the experiment of Keren and Roelofsma (1995), $82 \%$ of the subjects opt for 100 now and $63 \%$ for $\$ 110$ in 30 weeks. Subsequently, Keren and Roelofsma (1995) proposed the following choices (in all cases the lotteries pay zero with the residual probability):

Choice 3. 100 now with probability 0.9 or 110 in 4 weeks with probability 0.9

Choice 4. 100 in 26 weeks with probability 0.9 or 110 in 30 weeks with probability 0.9

In this case, the percentage of individuals choosing the immediate reward drops to $54 \%$ and it is further reduced to $39 \%$ in a version of the experiment with a lower probability of 0.5. Whereas the percentage of subjects choosing 110 in 30 weeks remains quite stable regardless of the probabilities. They claim that the main difference between the future and the present is an intrinsic uncertainty of the former. If subjects attach more utility to certainty (the present), the present bias occurs. When the present becomes uncertain, this distinction is no longer valid.

UDEU can capture a similar behavioral pattern in our Savagean uncertainty setting. Indeed, if we introduce a particular form of uncertainty in the immediate payoff, the present bias and diminishing impatience disappear. Indeed, conditional on each state, UDEU discounts the future exponentially, hence it is dynamically consistent. Let denote $(x, \omega, t)$ the 
following act (assuming $u(0)=0$ ):

$$
(x, \omega, t)= \begin{cases}x & \text { if }(\omega, t) \in \Omega \times \mathbb{T} \\ 0 & \text { otherwise }\end{cases}
$$

It is an act paying $x$ at time $t$ and state $\omega$ and zero otherwise, alternatively it can be written in the previous notation as $x_{\omega, t} 0$. It is the counterpart of Keren and Roelofsma (1995)'s lotteries in our setting.

Now, suppose that an individual is indifferent between $(x, \omega, t)$ and $(y, \omega, t+1)$ for some $y, x \in[w, b]$ and $t \in \mathbb{T}$. Then we have the following:

Fact 1. For all $x, y \in[w, b]$ and $t, t^{\prime} \in \mathbb{T}$.

$$
(x, \omega, t) \sim(y, \omega, t+1) \Longleftrightarrow\left(x, \omega, t^{\prime}\right) \succcurlyeq\left(y, \omega, t^{\prime}+1\right)
$$

Therefore, introducing a precise form of uncertainty cancels DI and the present bias. Since uncertainty resolves immediately, Fact 1 can be compared to the results of Keren and Roelofsma (1995). The explanation is similar, the main difference between the present and the future is the intrinsic uncertainty of the latter. In UDEU, the uncertainty of the future is modelled through uncertainty of the discount rate, since the present payoff is not discounted, it is certain (when state-independent). Differently, future payoffs, even the state-independent ones, are invariably uncertain. The additional impatience at certainty is captured by the inequality $I(0)>I(t)$ for all $t<T-1$ (when there are at least two different discount rates with strictly positive probability) or equivalently

$$
\frac{1}{E_{p}\left[\delta_{\omega}\right]}>\frac{E_{p}\left[\delta_{\omega}^{t}\right]}{E_{p}\left[\delta_{\omega}^{t+1}\right]}
$$

When we introduce uncertainty in the immediate payoff, the present loses its additional value and the individual behaves, conditionally to the state of nature, consistently. This is not only descriptively but also normatively appealing. If the individual knows the state of the world that will realize, she should behave consistently, being uncertainty the only force that induces inconsistency. 


\section{Consistent choice}

Dynamic inconsistent behavior may be prevented through commitment. There are various commitment devices (see Brocas et al., 2004), however, the effect of commitment is strongly related to the force behind the inconsistency. In the case of lack of self control, commitment devices such as, illiquid accounts, contracts or promises may be effective. Differently, under uncertainty, the effect of commitment made before uncertainty is resolved, may be detrimental. First, we have to clarify the relation between DI and of dynamic inconsistency under uncertainty. Consider an UDEU in which at time zero, the individual prefers $(100,0) \succcurlyeq(110,4)$, and $(110,30) \succcurlyeq(100,26)$. This preference can be dynamically inconsistent if at time $t=26$, the individual faces the same uncertainty of today, i.e she uses the same subjective probability at time 26. This is equivalent to assume i.i.d. uncertainty, in that case, there is no possibility of learning.

Going back to the example, at time zero, the individual prefers $(100,0) \succcurlyeq(110,4)$. Suppose she can commit to have the same preference for delayed payments, that is $(100,26) \succcurlyeq$ $(110,30)$, so she prevents the dynamic inconsistency (assuming i.i.d. uncertainty). Now suppose that $\omega \in \Omega$ realizes an that $\beta_{\omega}=0.98$, then, after uncertainty is resolved, she strictly prefers 110 in 4 weeks to 100 now and 110 in 30 weeks to 100 in 26 weeks. Therefore, the individual may ex-ante commit to a choice that she would revise if possible. Let's define the value of ex-ante commitment formally:

Definition 4. Ex-ante commitment is always valuable if, for all state-independent acts $x, y \in$ $\mathscr{H}$,

$$
\left(x_{0}, x_{1}, \ldots, x_{T}\right) \succcurlyeq\left(y_{0}, y_{1}, \ldots, y_{T}\right) \Longrightarrow\left(x_{0}, x_{1}, \ldots, x_{T}\right) \succcurlyeq_{\omega}\left(y_{0}, y_{1}, \ldots, y_{T}\right) \forall \omega \in \Omega
$$

Commitment is always valuable if ex-ante and all the conditional preferences agree on ranking the state-independent acts. The next theorem shows that ex-ante commitment is always valuable only under discounted expected utility. Intuitively, if there are two distinct discount rates, one can always find two acts that are ranked differently according to the conditional preferences, therefore making impossible ex-ante commitment to be always valuable.

Theorem 5. Ex-ante commitment is always valuable, if and only if, there is no uncertainty 
in the discount rate.

The conditions for ex-ante commitment to be always valuable is strong and indeed, it is satisfied only under discounted expected utility. The result raises the question of how to design effective commitment devices under uncertainty.

\section{Conclusion}

We introduced a model of intertemporal choice that takes into account the intrinsic uncertainty of the future through uncertainty in the discount rate. The model is behaviorally founded on a simple generalization of the discounted expected utility, uncertainty of the future is fully subjective. The model can account for diminishing impatience without invoking psychological factors. Commitment is valuable only if the discount rate is not uncertain and there is no learning about the structure of the underlying uncertainty. 


\section{A Appendix. Proofs}

We begin with a well known representation theorem.

Theorem Al (Köbberling and Wakker (2003)). Suppose there are at least two non-null states. Axioms Utility and T-OC are satisfied, if and only if, there exists a utility $V$ representing $\succcurlyeq$ of the form

$$
V(c)=\sum_{(\omega, t)} u(c(\omega, t)) p(\omega, t)
$$

where $p$ is a unique probability and $u$ is a strictly increasing and cardinally unique utility function.

Proof. Of Theorem 1. Given Theorem A1, let's rewrite $V(c)$ in the following form:

$$
V(c)=\sum_{\omega \in \Omega} p(\omega) \sum_{t \in \mathbb{T}} \delta^{(t \mid \omega)} u(c(\omega, t))
$$

where $\delta^{(t \mid \omega)}$ is the conditional probability on $\mathbb{T}$ given $\omega \in \Omega$, and $p$ is the marginal. The fact that Conditional Stationarity implies $\delta^{(t \mid \omega)}=\delta_{\omega}^{t}$ for some $\delta_{\omega}>1$ follows from standard argument (for example Koopmans (1960)).

Proof. Of Theorem 5. W.l.o.g. let assume $u([w, b]) \supseteq[-1,1]$ and that $u(0)=0$. If there is no uncertainty in the discount, than UDEU collapses to discounted EU, so the result is trivial. Assume commitment is always valuable and assume w.l.o.g. the existence of $\delta_{\omega}>\delta_{\omega^{\prime}}$. Then, take $x \in u([w, b])$ with $\delta_{\omega}>u(x)>\delta_{\omega^{\prime}}$. Equivalently we have $0>u(x)-\delta_{\omega}$ and $0<u(x)-\delta_{\omega^{\prime}}$. These two inequalities implies that the act $(0,0, \ldots, 0)>_{\omega}(x, z, 0, \ldots, 0)$ and $(x, z, 0, \ldots, 0)>_{\omega^{\prime}}(0,0, \ldots, 0)$ for $z \in[w, b]$ with $u(z)=-1$. By completeness of the unconditional preference, either $(0,0, \ldots, 0) \succcurlyeq(x, z, 0, \ldots, 0)$ or $(x, z, 0, \ldots, 0) \succcurlyeq(0,0, \ldots, 0)$, a contradiction to the fact that ex-ante commitment is always valuable.

\section{References}

Azfar, O. (1999). Rationalizing hyperbolic discounting. Journal of Economic Behavior \& Organization 38(2), 245-252.

Benzion, U., A. Rapoport, and J. Yagil (1989). Discount rates inferred from decisions: An experimental study. Management Science 35(3), 270-284.

Brocas, I., J. Carrillo, and M. Dewatripont (2004). Commitment devices under self-control problems: an overview. In I. Brocas and J. Carrillo (Eds.), The Psychology of Economic Decisions. Volume 2: Reasons and Choices. Oxford: Oxford University Press.

Dasgupta, P. and E. Maskin (2005). Uncertainty and hyperbolic discounting. American Economic Review 95, 1290-1299.

Epper, T. and H. Fehr-Duda (2014). The missing link: unifying risk taking and time discounting. Mimeo (June).

Farmer, J. D. and J. Geanakoplos (2009). Hyperbolic discounting is rational: Valuing the far future with uncertain discount rates. Technical Report 1719, Cowles Foundation for Research in Economics, Yale University. 
Fisher, I. (1930). The theory of interest. New York: Macmillan.

Halevy, Y. (2008). Strotz meets Allais: Diminishing impatience and the certainty effect. American Economic Review 98(3), 1145-62.

Harris, C. and D. Laibson (2013). Instantaneous gratification. The Quarterly Journal of Economics 128(1), 205-248.

Hayashi, T. (2003). Quasi-stationary cardinal utility and present bias. Journal of Economic Theory 112(2), $343-352$.

Higashi, Y., K. Hyogo, and N. Takeoka (2009). Subjective random discounting and intertemporal choice. Journal of Economic Theory 144(3), 1015-1053.

Jackson, M. O. and L. Yariv (2012). Present bias and collective dynamic choice in the lab. Available at SSRN 2161036.

Karni, E. and I. Zilcha (2000). Saving behavior in stationary equilibrium with random discounting. Economic Theory 15(3), 551-564.

Keren, G. and P. Roelofsma (1995). Immediacy and certainty in intertemporal choice. Organizational Behavior and Human Decision Processes 63(3), 287 - 297.

Köbberling, V. and P. Wakker (2003). Preference foundations for nonexpected utility: a generalized and simplified technique. Mathematics of operation research 28(3), 395-423.

Koopmans, T. C. (1960). Stationary ordinal utility and impatience. Econometrica 28, 287.

Laibson, D. (1997). Golden eggs and hyperbolic discounting. The Quarterly Journal of Economics 112(2), 443-77.

Loewenstein, G. and D. Prelec (1992). Anomalies in intertemporal choice: Evidence and an interpretation. The Quarterly Journal of Economics 107(2), 573-97.

Luttmer, E. G. J. and T. Mariotti (2003). Subjective discounting in an exchange economy. Journal of Political Economy 111(5), 959-989.

Montiel Olea, J. L. and T. Strzalecki (2014). Axiomatization and measurement of quasihyperbolic discounting. The Quarterly Journal of Economics, Forthcoming.

Myerson, J., L. Green, J. Scott Hanson, D. D. Holt, and S. J. Estle (2003). Discounting delayed and probabilistic rewards: Processes and traits. Journal of Economic Psychology 24(5), 619-635.

Prelec, D. (2004). Decreasing impatience: A criterion for non-stationary time preference and "hyperbolic" discounting. Scandinavian Journal of Economics 106(3), 511-532.

Saito, K. (2011). A relation between risk and time preferences. Mimeo (Feb.).

Shane, F., G. Loewenstein, and T. O'Donoghue (2002). Time discounting and time preference: A critical review. Journal of Economic Literature 40(2), 351-401.

Sozou, P. D. (1998). On hyperbolic discounting and uncertain hazard rates. Proceedings of the Royal Society of London. Series B: Biological Sciences 265(1409), 2015-2020.

Strotz, R. H. (1956). Myopia and inconsistency in dynamic utility maximization. The Review of Economic Studies 23(3), 165-180.

Thaler, R. (1981). Some empirical evidence on dynamic inconsistency. Economics Letters 8(3), 201-207.

Weber, J. B. and G. B. Chapman (2005). The combined effects of risk and time on choice: Does uncertainty eliminate the immediacy effect? Does delay eliminate the certainty effect? Organizational Behavior and Human Decision Processes 96(2), $104-118$.

Weitzman, M. L. (1998). Why the far-distant future should be discounted at its lowest pos- 
sible rate. Journal of Environmental Economics and Management 36(3), 201 - 208.

Yaari, M. E. (1965). Uncertain lifetime, life insurance, and the theory of the consumer. The Review of Economic Studies 32(2), 137-150. 